\title{
Physical Phase Plates for Cryo-Electron Microscopy of Biological Specimens: Comparison of Hole-Free Phase Plates and Zach Electrostatic Phase Plates
}

\author{
Martin Obermair ${ }^{1}$, Michael Marko², Simon Hettler ${ }^{1}$, Chyongere Hsieh², Dagmar Gerthsen ${ }^{1}$ \\ 1. Laboratory for Electron Microscopy, Karlsruhe Institute of Technology, Karlsruhe, Germany. \\ 2. Wadsworth Center, New York State Department of Health, Albany NY, USA.
}

The weak contrast of life-science specimens in (cryo-) transmission electron microscopy (TEM) has led to a strong interest in the development of physical phase plates (PPs). By inducing a relative phase shift between scattered and unscattered electrons in the back focal plane (BFP) of the objective lens, PPs enable the generation of phase contrast without the need to apply a strong defocus. A large number of PP concepts exist [1], which can be mainly divided into thin-film and electrostatic approaches.

We present results achieved with an electrostatic Zach PP [2] and with a hole-free phase plate (HFPP) [3-5]. The HFPP exploits the formation of a charged patch, which is induced by the intense zero-order beam on a thin amorphous-carbon film; the patch shifts the phase of the unscattered beam. However, the application of thin-film PPs is limited due to the fixed phase shift value and the scattering of electrons in the PP material. These limitations do not exist for electrostatic PPs, where a strongly localized electrostatic potential induces an adjustable phase shift on the unscattered electrons. The electrostatic Zach PP consists of a single rod carrying an isolated and shielded open gold electrode (Figure 1). When a voltage is applied to the electrode, an inhomogeneous electrostatic potential is formed at the tip. For the presented experiments, the HFPP was placed in the BFP of a JEOL JEM-3200FSC/PP, operated at $300 \mathrm{kV}$ and the Zach PP was placed in a conjugate BFP by the use of a transfer lens.

To compare phase-contrast imaging of these two types of PPs, image series and cryo-tomograms of amorphous-ice-embedded cryo-TEM samples such as T4 bacteriophages (T4), ribosomes and tobacco mosaic virus were recorded. Figure 2 shows application of a Zach PP for a bacteriophage T4 sample. By applying a negative potential of $-5 \mathrm{~V}$ to the Zach PP (Fig. 2a), there is strong contrast enhancement of the 250-nm T4 head (yellow arrow) and tail (green arrow), compared to imaging without an applied voltage (Fig. 2b). A potential of $+8 \mathrm{~V}$ (Fig. 2c) results in contrast inversion, which is strongly visible in the T4 and in the gold nanoparticles (red arrow). Figure 3 shows the application of a HFPP to the T4 sample, both images recorded in focus. Line scans demonstrate the contrast enhancement of a T4 head with and without the HFPP (Figs. $2 \mathrm{a}$ and $2 \mathrm{~b}$ ). This is further illustrated by the intensity-line profiles in Fig. 2c, which also shows a reduction of the background grey value, due to electron scattering in the HFPP. In summary, comparable contrast enhancement is achieved with the two PP types, and both approaches exhibit advantages and drawbacks [6].

References:

[1] R M Glaeser, Rev. Sci. Instrum. 84 (2013), p. 111101.

[2] S Hettler et al, Microsc Microanal 18 (2010), p. 1010.

[3] M Marko et al, Microsc. Microanal. 22 (2016), p. 1316.

[4] M Malac et al, Ultramicroscopy 118 (2012), p. 77.

[5] R Danev et al, PNAS 111 (2014), p. 15635.

[6] Funding from the Deutsche Forschungsgemeinschaft (DFG) and the Karlsruhe House of Young Scientists (KHYS) and from NIH grant R35GM119023 (to MM) is acknowledged 

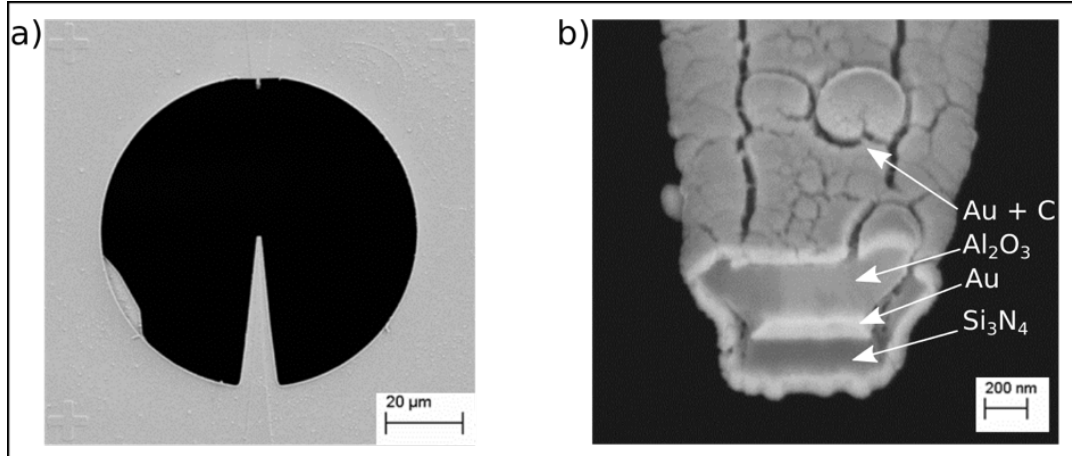

Figure 1. Scanning electron microscopy images of the Zach PP used for this work. (a) Overview image shows the round aperture with the single rod. (b) The layer system of the Zach PP is visible in the image of the open tip of the PP rod.
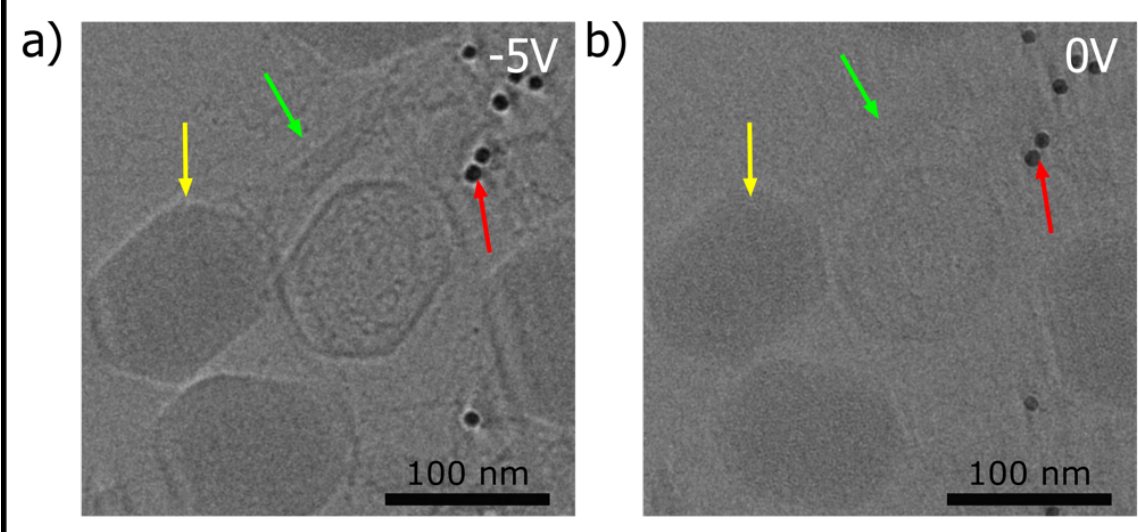

c)

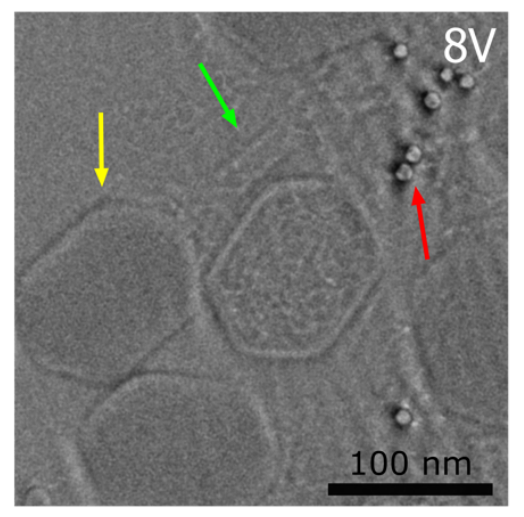

Figure 2. Zach PP TEM images of a T4 sample. (a-c). Images of a voltage series with $-5 \mathrm{~V}, 0 \mathrm{~V}$ and 8 $\mathrm{V}$ applied to the Zach PP electrode, showing dark contrast for negative voltage and bright contrast for positive voltage.

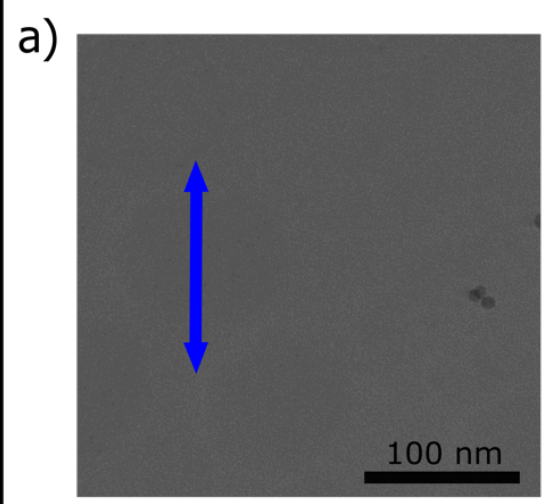

b)

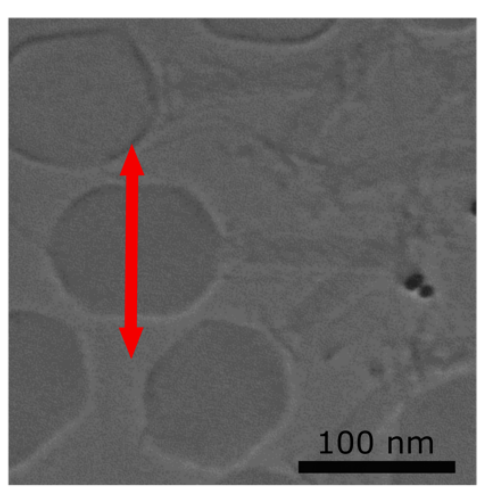

c)

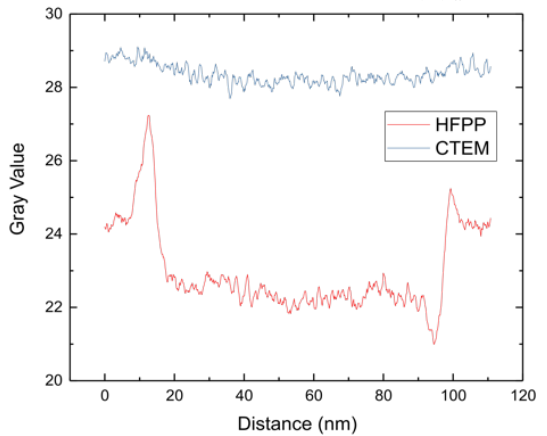

Figure 3. In-focus images of a T4 sample obtained with (a) conventional TEM (CTEM) and (b) using a HFPP. Line scans across T4 head in (a) and (b) are shown in (c). 